\title{
Fuzzy logic applied to value of information assessment in oil and gas projects
}

\author{
Martin Vilela ${ }^{1} \cdot$ Gbenga Oluyemi $^{1} \cdot$ Andrei Petrovski $^{2}$
}

Received: 20 July 2018 / Published online: 3 July 2019

(c) The Author(s) 2019

\begin{abstract}
The concept of value of information (VOI) has been widely used in the oil industry when making decisions on the acquisition of new data sets for the development and operation of oil fields. The classical approach to VOI assumes that the outcome of the data acquisition process produces crisp values, which are uniquely mapped onto one of the deterministic reservoir models representing the subsurface variability. However, subsurface reservoir data are not always crisp; it can also be fuzzy and may correspond to various reservoir models to different degrees. The classical approach to VOI may not, therefore, lead to the best decision with regard to the need to acquire new data. Fuzzy logic, introduced in the 1960s as an alternative to the classical logic, is able to manage the uncertainty associated with the fuzziness of the data. In this paper, both classical and fuzzy theoretical formulations for VOI are developed and contrasted using inherently vague data. A case study, which is consistent with the future development of an oil reservoir, is used to compare the application of both approaches to the estimation of VOI. The results of the VOI process show that when the fuzzy nature of the data is included in the assessment, the value of the data decreases. In this case study, the results of the assessment using crisp data and fuzzy data change the decision from "acquire" the additional data (in the former) to "do not acquire" the additional data (in the latter). In general, different decisions are reached, depending on whether the fuzzy nature of the data is considered during the evaluation. The implications of these results are significant in a domain such as the oil and gas industry (where investments are huge). This work strongly suggests the need to define the data as crisp or fuzzy for use in VOI, prior to implementing the assessment to select and define the right approach.
\end{abstract}

Keywords Value of information · Fuzzy logic $\cdot$ Uncertainty and risk management · Oil and gas industry

$\begin{array}{ll}\text { Abbreviations } \\ \text { EV } & \text { Expected value } \\ \text { IOR } & \text { Initial oil rate } \\ \text { US\$ } & \text { US dollars } \\ \text { OCT } & \text { Oil column thickness } \\ \text { VOI } & \text { Value of information }\end{array}$

Edited by Xiu-Qin Zhu

Martin Vilela

vilelamartin1@yahoo.es; m.j.vilela-ibarra@rgu.ac.uk

1 School of Engineering, Robert Gordon University, Garthdee Road, Aberdeen AB10 7QB, Scotland, UK

2 School of Computing, Robert Gordon University, Garthdee Road, Aberdeen AB10 7QB, Scotland, UK

\section{Introduction}

Decision-making is a central process in any business, and decisions are routinely made in the oil and gas industry that could impact on the business in the short, medium or long term. A key element for making consistent and robust decisions is to use a prescriptive method for assessing each alternative option (Howard 1966; Raiffa 2007). The need to use such methods is even stronger when the variables involved in the decision carry uncertainties.

The types of decisions explored in this paper are those associated with data acquisition and, more specifically, data acquisition in the context of subsurface evaluations in the oil and gas industry. In the subsurface domain, the variables defining a reservoir and its production capability (permeability, fluid contact, capillary pressure, etc.) are subject to uncertainty due to randomness and imprecision. The uncertainty in these variables results in an uncertainty in the reservoir production forecast, making it difficult to assess the 
financial benefits of developing the field or even to decide on whether to perform tasks to optimise hydrocarbon production. The uncertainty in the project outcome involves the risk of financial losses, which need to be avoided.

The uncertainty refers to our knowledge of the input parameters and, consequently, of the outcomes of the system; certainty measures our degree of knowledge, and probability is the tool used to describe the chance that a particular value of a parameter will be obtained.

In most cases, data can be acquired to enable a better understanding of the uncertainties with the aim of reducing them. However, the value of the acquired data is not measured by the reduction in uncertainty but by the reduction of risk and an increase in the project's value. VOI is the methodology developed to assess data acquisition decisions in uncertain conditions, which is typically the case for subsurface projects.

Works in the field of decision-making for data acquisition were pioneering studies by Grayson (1960), Raiffa and Schlaifer (1961) and Newendorp (1967). Subsequently, further research and applications expanded the scope of the subject and provided more robustness in the methodology. Warren (1983) discussed a methodology for deciding between initiating or rejecting a project and deferring the decision until more information is acquired, using a field development decision as an example. Lohrenz (1988) presented four examples in the petroleum engineering domain, using decision trees in order to evaluate data acquisition. Demirmen (1996) used VOI methodology to justify and rank subsurface appraisal projects. Newendorp and Schuyler (2002) developed fundamental ideas related to VOI, including examples from the exploration and appraisal of oil and gas projects. Koninx (2000) discussed VOI from a methodological perspective, adding examples related to the value of 3D seismic acquisition and appraisal to clearly define the hydrocarbon composition. In other research, Coopersmith and Cunningham (2002) proposed a step-wise methodology to facilitate VOI assessment and, through SPE-related publications, Bratvold et al. (2007) showed that although the use of systematic qualitative methods in VOI has increased in recent years, it is still far from being a standard application, even when large investments are involved. Begg et al. (2002) introduced alternative concepts for assessing the value of an uncertain project, such as the value of flexibility, a complementary methodology to VOI. Kullawan et al. (2014) discussed an important application of VOI in a geosteering operation in which a large number of real-time operations are executed day to day, demonstrating the flexibility of the VOI methodology in adapting to challenging circumstances. Bickel (2014) applied decision analytics and value of information for unconventional reservoirs. Ferreira (2015) discussed a probabilistic approach to quantify the value of information associated with a 4D seismic acquisition project. Steineder et al. (2018) used the value of information methodology to assess a pilot project polymer injection in a horizontal well. Grose and Smalley developed a risk-based surveillance planning method based on a value of information approach for data acquisition in producing fields (2017). Shabair et al. (2017) discussed a practical implementation of value of information applied to a reservoir surveillance plan for a fractured carbonate under waterflooding. Similarly, Clemen (1996) and Suslick and Schiozer (2004) discussed applications and methods which enrich the VOI process.

All of these works contributed to the development of a robust and consistent theory for assessing the value of data gathering, with specific applications to the oil and gas industry. The research assumes that the data acquired will produce accurate information, which means that the remaining uncertainty is only that which is caused by randomness. However, in subsurface projects in the oil and gas industry, there are cases in which data are not crisp but fuzzy, and this imprecision in the aggregated data determines the project's value. If the data proposed to be acquired are fuzzy, a methodology that assesses the value associated with data acquisition must include the fuzziness of the data unless the proven form of this uncertainty (fuzziness) leaves the project's value unchanged. Fuzzy logic has been applied in recent years to several problems related to the oil and gas industry, such as Ahmed et al. (2019) in the prediction of the rate of penetration during drilling in a shale formation, Sari (2016) for estimating the rock strength, Thong and Kepic (2015) developing fuzzy clustering techniques to incorporate prior information into seismic impedance inversion, Nashawi and Malallah (2010) making a permeability prediction from wireline well logs using fuzzy logic, Ebrahimi and Sajedian (2010) developing a method for predicting the two-phase inflow performance relationship of horizontal oil wells. Meanwhile, Ivanovich et al. (2012) used a fuzzy petrophysical compositions method for calculating hydrocarbon reserves, Popa (2013) discussed the identification of horizontal well placement using fuzzy logic, Bermudez et al. (2014) developed a methodology for the monitoring and prediction of unexpected behaviour in electric submersible pumps, and Mirzabozorg et al. (2014) implemented a methodology for the incorporation of engineering knowledge during history matching performance prediction using fuzzy logic. Nageh et al. (2015) developed a screening criterion for EOR technologies using fuzzy logic as an artificial intelligence technique, Bukhamseen et al. (2016) built a streamlined model guided by fuzzy logic to optimise field injection and production strategies, Grassian et al. (2017) developed a fuzzy expert system for analysing and optimising submersible electric pump failure modes, and Passalacqua and Qubian (2018) implemented a decision-making approach for heavy oil field projects using a fuzzy analytical 
hierarchy process. The aim of this work is to integrate the fuzzy characteristics of subsurface data into the classical theory for valuing data gathering in order to develop a complete methodology for assessing the value of acquiring fuzzy data, as applicable to decisions being made in the oil and gas industry.

\section{Model formulation}

The first step in applying the classical VOI method is to define a set of $n$ discrete states of nature (known as "cases") $s_{1}, s_{2}, \ldots s_{n}$ that describe the range of all possible project outcomes. Each state has a probability of occurrence $p\left(s_{i}\right)$ where (Clemen 1996):

$$
\sum_{i=1}^{n} p\left(s_{i}\right)=1
$$

The probabilities in Eq. (1) are known as "prior probabilities" because they represent the current belief (i.e. before the acquisition of new data) regarding the likelihood that a state will occur. Experts assign these probabilities based on their experience and judgment.

We now assume a decision problem with $m$ alternative solutions included in the set $A$ :

$A=\left\{a_{1}, a_{2}, a_{3}, \ldots, a_{m}\right\}$

For each pair (the alternative $a_{j}$ and the state of nature $s_{i}$ ), there is a value $u_{j i}$, which is the value that will materialise in the future if the alternative $a_{j}$ and state $s_{i}$ occur.

The expected value (EV) corresponding to the $j$ th alternative is defined as:

$\mathrm{EV}\left(a_{j}\right)=\sum_{i=1}^{n} u_{j i} p\left(s_{i}\right)$

Typically, the decision criterion used is to select the alternative with the maximum $\mathrm{EV}$ :

$\mathrm{EV}\left(a^{*}\right)=\underset{j}{\max \operatorname{EV}\left(a_{j}\right)}$

Equation (4) represents the value of the project without information (i.e. with the actual information) which, in the subsurface domain, typically includes several uncertainties in the input parameters that, in turn, will result in uncertainties in the outcomes.

There are situations in which additional data may be acquired (in the future) that could narrow the uncertainty in the input parameters that are responsible for the spread (uncertainties) in the outcomes. Acquiring these data would affect the value of each discrete state and would also modify the probabilities assigned to each state. The net effect of the changes to the values and probabilities of the states (cases) is a change in the project's value.

In general (Bratvold et al. 2007):

$\mathrm{VOI}=\mathrm{EV}_{\text {with information }}-\mathrm{EV}_{\text {without information }}$

Both values, $\mathrm{EV}_{\text {with information }}$ and $\mathrm{EV}_{\text {without information }}$, contain what we believe the outcome of the project would be in two different situations, both of which are in the future.

Let us assume that the outcomes resulting from the acquired data are discretised in the following set $X$ of $l$ values:

$X=\left\{x_{1}, x_{2}, \ldots, x_{l}\right\}$

Here, the elements of the set $X, x_{1}, x_{2}, \ldots, x_{l}$ are the values measured or estimated during the data acquisition process; they can be values of porosity, permeability, pressure, depth, etc. (in their corresponding units). The reliability probabilities $p\left(x_{k} \mid s_{i}\right)$ are assigned by experts in the same way as the prior probabilities in Eq. (1). The reliability probabilities measure the likelihood that the data accurately identifies the states of nature. Because data are imperfect in the real world, reliability probabilities are always less than 1 . In the Bayesian inference system, imperfect data are the opposite to perfect data, which is an ideal (not a real-world) concept and assumes that data can accurately predict the state of nature.

Reliability probabilities are flipped using Bayes' theorem to generate the posterior probabilities, as in Eq. (7):

$p\left(s_{i} \mid x_{k}\right)=\frac{p\left(x_{k} \mid s_{i}\right) p\left(s_{i}\right)}{p\left(x_{k}\right)}$

The denominator in Eq. (7) is the marginal probability of the new data $p\left(x_{k}\right)$, which is defined using the total probability theorem, as given in Eq. (8):

$p\left(x_{k}\right)=\sum_{i=1}^{n} p\left(x_{k} \mid s_{i}\right) p\left(s_{i}\right)$

Given the data outcome $x_{k}$, the EV for the $j$ th alternative is:

$\operatorname{EV}\left(u_{j} \mid x_{k}\right)=\sum_{i=1}^{n} u_{j i} p\left(s_{i} \mid x_{k}\right)$

$\mathrm{EV}\left(u_{j} \mid x_{k}\right)$ is the expected value of the project for the $j$ th alternative and the data outcome $x_{k}$.

The optimum alternative is that which maximises the EV:

$\operatorname{EV}\left(a^{*} \mid x_{k}\right)=\underset{j}{\max \operatorname{EV}\left(a_{j} \mid x_{k}\right)}$

The unconditional maximum EV (i.e. the EV of the project taking into account the data acquisition outcomes) is the 
sum of the conditional EV weighted with the corresponding marginal probabilities:

$\mathrm{EV}\left(a_{x}^{*}\right)=\sum_{k=1}^{m} \operatorname{EV}\left(a^{*} \mid x_{k}\right) p\left(x_{k}\right)$

Finally, the VOI is the difference between the EV of the project with information and the $\mathrm{EV}$ of the project without information (Bratvold et al. 2007), given in Eqs. (11) and (4):

$\mathrm{VOI}=\mathrm{EV}\left(a_{x}^{*}\right)-\operatorname{EV}\left(a^{*}\right)$

In the oil and gas industry, uncertainty may be the result of lack of information, inaccuracy of measurements or lexical vagueness. Typical examples of lack of information are the porosity and permeability values used to populate the reservoir models between the existing wells. Probability techniques are used for managing uncertainties due to incomplete information. Inaccuracy of measurement relates to the measuring tools used and to the classification and interpretation of the measurement. For example, pressure gradient measurements have uncertainties associated with the measured value and depth; similarly, the saturation values of the remaining oil usually carry uncertainties related to the methods, conditions and accuracy of the logs and laboratory experiments. The subjectivity of the interpreter also adds uncertainty to the values resulting from the data: the interpretation of results often results in categories with lexical vagueness such as "large", "profitable" and "small". These uncertainties, due to inaccuracy of measurement and lexical vagueness, introduce imprecision in the data. In this paper, we show how fuzzy logic can be used to manage uncertainty in VOI assessment in the oil and gas industry.

There are two approaches for understanding the outcome of data acquisition: crisp data and fuzzy data. In the crisp approach, the outcome of the data acquisition falls into only one of the discrete intervals into which the range of possible outcomes of the data acquisition is divided, while in the fuzzy approach (fuzzy VOI), the outcome of the data may fall into more than one of these discrete intervals. A discussion of the latter approach is given in the remainder of this section.

Fuzzy logic was introduced by Zadeh (1965) to deal with the uncertainty associated with vagueness and imprecision in data; further developments by Zadeh (1968), Okuda (1978), Hayward and Davidson (2003) and Sivanandam et al. (2010) gave rise to a complete logic system that is applicable in many domains.

In classical logic, an element or event either "belongs" to a set of outcomes or does not and this is described using the binary representation of zero or one. In classical set theory, this is referred to as the characteristic function for the set of events. Fuzzy logic extends the concept of the characteristic function to a membership function, which represents the meaning of "belonging" as a continuous value between zero and one. In this way, the degree to which an event belongs to the sets of outcomes is represented by the membership function of that event on those sets.

Fuzzy logic captures vagueness through the membership function, which is a mapping from a given universe of discourse $X$ to a unit interval containing the membership values.

In a crisp set of events $M$, the probability of occurrence of the events in the set is:

$P(M)=\sum_{x \in X} p(x) \mu_{M}=\sum_{x_{k} \in M} p\left(x_{k}\right)$

where $M \subset X$.

In Eq. (13), $p(x)$ is the probability of the occurrence of event $x, \mu_{M}$ is the characteristic function [defined in Eq. (15)], and $p\left(x_{k}\right)$ are the probabilities of the events where the characteristic function is 1 .

The characteristic function is (Zadeh 1965):

$\mu_{M}=\left\{\begin{array}{l}1, \quad x_{k} \in M \\ 0, \text { otherwise }\end{array}\right.$

For a fuzzy set, the probability of a fuzzy event $\tilde{M}$ is:

$P(\tilde{M})=\sum_{k=1}^{r} \mu_{\tilde{M}}\left(x_{k}\right) p\left(x_{k}\right)$

where $\mu_{\tilde{M}}\left(x_{k}\right)$ is the membership function $\mu_{\tilde{M}}$ evaluated for the value $x_{k}$.

The posterior probabilities of the states of nature, given the fuzzy event $\tilde{M}$, are given by Eq. (17), assuming that the reliability, prior probabilities and membership functions of the fuzzy events are known (Ross 2010):

$P\left(s_{i} \mid \tilde{M}\right)=\frac{\sum_{k=1}^{r} p\left(x_{k} \mid s_{i}\right) \mu_{\tilde{M}}\left(x_{k}\right) p\left(s_{i}\right)}{P(\tilde{M})}=\frac{P\left(\tilde{M} \mid s_{i}\right) p\left(s_{i}\right)}{P(\tilde{M})}$

where the fuzzy reliability probabilities are:

$P\left(\tilde{M} \mid s_{i}\right)=\sum_{k=1}^{r} p\left(x_{k} \mid s_{i}\right) \mu_{\tilde{M}}\left(x_{k}\right)$

An orthogonal fuzzy system is a set $\emptyset$ of fuzzy sets, $\emptyset=\left\{\tilde{M}_{1}, \tilde{M}_{2}, \ldots \tilde{M}_{l}\right\}$, satisfying the condition that:

$\sum_{f=1}^{l} \mu_{\tilde{M}_{f}}\left(x_{m}\right)=1 \quad\left\{\right.$ for all $\left.x_{m} \in X\right\}$

For fuzzy events, if the fuzzy system is an orthogonal set and the data outcome is represented by the fuzzy set $\tilde{M}_{k}$, 
the $\mathrm{EV}$ of the $j$ th alternative and membership function $\tilde{M}_{f}$ is given by:

$\operatorname{EV}\left(a_{j} \mid \tilde{M}_{f}\right)=\sum_{i=1}^{n} a_{i j} p\left(s_{i} \mid \tilde{M}_{f}\right)$

The optimum alternative given the fuzzy set $\tilde{M}_{k}$ is that which maximises the EV:

$\operatorname{EV}\left(a^{*} \mid \tilde{M}_{f}\right)=\max _{j} \operatorname{EV}\left(a_{j} \mid \tilde{M}_{f}\right)$

The unconditional maximum EV takes the form:

$\operatorname{EV}\left(a_{\emptyset}^{*}\right)=\sum_{f=1}^{l} \operatorname{EV}\left(a^{*} \mid \tilde{M}_{f}\right) p\left(\tilde{M}_{f}\right)$

Finally, the VOI is the difference between the EV with information and the EV without information, Eqs. (22) and (4):

$\mathrm{VOI}=\operatorname{EV}\left(a_{\emptyset}^{*}\right)-\operatorname{EV}\left(a^{*}\right)$

In summary, the four key equations discussed in this section are listed in Table 1.

\section{VOI case study: classical versus fuzzy approaches}

The value of projects with uncertain values may be affected when additional information regarding the input variables is gathered. The reliability probability of the data, which measures the likelihood that new data could accurately identify the states of nature, is estimated based on expert experience and judgment. Once the reliability probability has been estimated, Bayes' theorem is used to invert the reliability probabilities to generate the posterior probabilities, which are then used to compute the EV of the project with the new data.
In classical logic, there is a set of mutually exclusive discrete events that expand the range of possible outcomes of the data acquisition; the outcome of a proposed data acquisition belongs to one of those events in a crisp manner. However, there are cases in which the outcome of the proposed data acquisition belongs to the discrete sets of events in a "vague" or "fuzzy" way; in these cases, changes in the VOI assessment must be made to accommodate these fuzzy data.

In the oil and gas industry (particularly in the subsurface domain), there are situations in which the data are vague or diffuse. However, there are no reported cases in the literature on the use of fuzzy data in assessing VOI.

The aim of this paper is to integrate the imprecision of the data in the VOI and assess its impact on a data acquisition decision in an oil field. The impact of the imprecision in the data is measured by comparing the results of VOI using crisp and fuzzy data.

\subsection{Case study: reservoir description}

In this section, an oilfield located in North Africa is used as a case study to evaluate a VOI problem where the data that may be acquired are fuzzy.

The project involves the exploitation of a sandstone oil field, composed of three isolated blocks of good quality rock with a thin hydrocarbon column. Two compartments with similar petrophysical properties (blocks A and B) have been drilled and produced using three vertical wells each. The wells are located in a pattern of one producer and one injector well. The reservoir section contains well-sorted grains with an average porosity of $25 \%$ and an average permeability of $250 \mathrm{mD}$. The oil column thickness (OCT) of the wells ranges between a minimum of $11 \mathrm{ft}$ and a maximum of $42 \mathrm{ft}$, with averages of $38 \mathrm{ft}$ and $16.1 \mathrm{ft}$ for blocks A and $\mathrm{B}$, respectively.

Blocks A and B are separated by a north-south fault with a throw of approximately $25 \mathrm{ft}$, which isolates the blocks from each other. Initially, the isolation of the blocks was an assumption based on geological hypotheses and analogue information, but it was later confirmed, based on production and injection data from the wells. Seismic vertical resolution

Table 1 Key equations discussed in the model formulation section

\begin{tabular}{lll}
\hline Serial number & Equation & Comments \\
\hline 5 & VOI $=\mathrm{EV}_{\text {with information }}-\mathrm{EV}_{\text {without information }}$ & Definition of VOI \\
12 & $\mathrm{VOI}=\operatorname{EV}\left(a_{x}^{*}\right)-\operatorname{EV}\left(a^{*}\right)$ & Crisp definition of VOI \\
23 & $\mathrm{VOI}=\operatorname{EV}\left(a_{\emptyset}^{*}\right)-\operatorname{EV}\left(a^{*}\right)$ & Fuzzy definition of VOI \\
17 & $P\left(s_{i} \mid \tilde{M}\right)=\frac{\sum_{k=1}^{r} p\left(x_{k} \mid s_{i}\right) \mu_{\tilde{M}}\left(x_{k}\right) p\left(s_{i}\right)}{P(\tilde{M})}=\frac{P\left(\tilde{M} \mid s_{i}\right) p\left(s_{i}\right)}{P(\tilde{M})}$ & $\begin{array}{c}\text { Definition of posterior prob- } \\
\text { abilities on the fuzzy logic } \\
\end{array}$ \\
& & frame \\
\hline
\end{tabular}


is between 20 and $30 \mathrm{ft}$, making it challenging to identify the top and base of the reservoir with enough accuracy to detect structural shifting in the wells.

Due to the nature of the reservoir and the performance of analogue reservoirs, each producer was drilled in patterns with a nearby injector well, to ensure pressure maintenance and sweep efficiency. In order to allow water to be injected without compromising the integrity of the cap rock, water injection commenced when the initial bottom-hole pressure in the producer wells fell by 1000 psi from the initial pressure (the approximate initial value was $2500-1500 \mathrm{psi}$ ), which typically occurs after 1 year of production.

\subsection{Reservoir performance}

Oil production from block A started eight and a half years ago (January 2010), and production from block B commenced 2 years later. Figures 1 and 2 show the historical, until 2018, as well as the predicted oil rates for the existing wells until 2029, when the 20-year concession licence expires. The wells show similar performance trends, the main difference being the initial oil production rates (IORs) of the wells for blocks A (Fig. 1) and B (Fig. 2).

The technical team working on this project found a correlation between the OCT and initial oil rate (IOR). Figure 3 shows the OCT and IOR measured in each well as well as the correlation found between these parameters.

The linear relationship between IOR and OCT shows that an increase in OCT correlates with an increase in IOR for each well.

To establish the relationship between IOR and OCT, we use a linear correlation; in the study case discussed in this paper, the data are limited to what was measured in the field, and the experts identified that the larger the OCT, the higher the IOR and realised that these two parameters seem to follow a linear trend. On the other hand, the relation between these parameters has a physical meaning through Darcy's law for the flow of fluids in porous media: in a horizontal

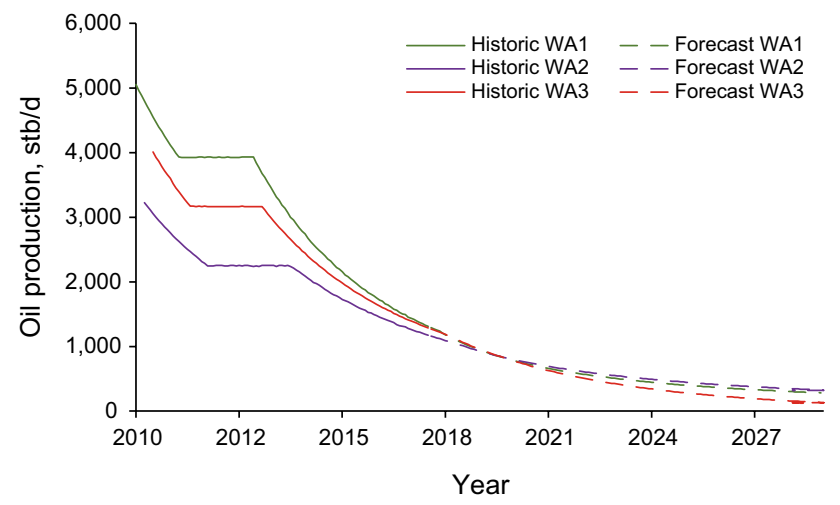

Fig. 1 Historic and predicted well performance—block A

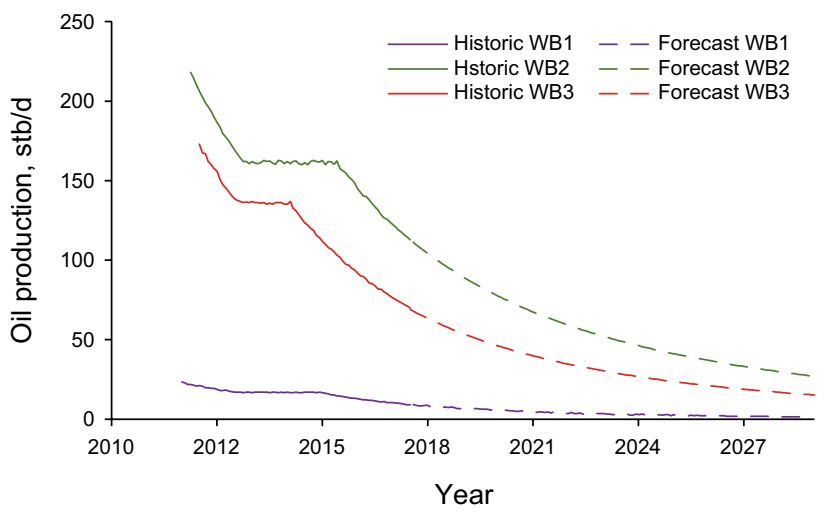

Fig. 2 Historic and predicted well performance-block B

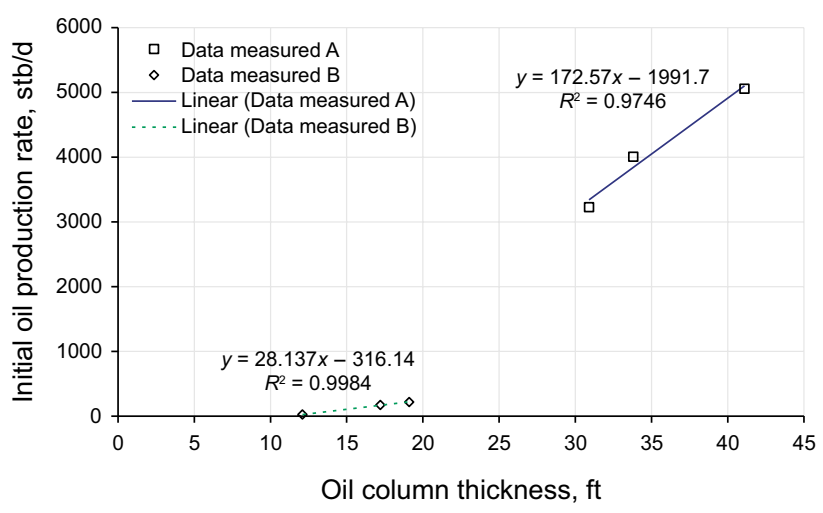

Fig. 3 Linear correlation between IOR and OCT for the wells in blocks A and B

linear medium, the flow is proportional to the cross-sectional area of the rock which is the product of thickness and width. The main limitation of the correlation is the small sample used, which, however, due to the support provided by Darcy's law, gives robustness to this approach.

This correlation has several drawbacks due to the uncertainties in the measured OCT and these include:

(1) Repeat formation tester (RFT) data failure. During operation of the RFT, there were repeated failures in gathering the pressure data points (due to seal failures or poor fluid mobility) and the data that were interpreted carried a great deal of ambiguity. Many data points do not fit into the water or oil profiles, and these results mean that the definition of OCT is ambiguous.

(2) Fuzzy OCT. Log interpretation does not show a crisp indication of OCT, and the interpreted water saturation response curve does not show a clear transition between the interval with $100 \%$ water saturation and the interval with irreducible water saturation. This ambiguity in the 
inter-fluid contact creates vagueness in the estimated values for OCT.

(3) Unclear definition of the limits of the transition zone. The limits of the transition zone are not clearly defined. It could be interpreted that the complete reservoir section is within the transition zone or that the tools used are not capable of distinguishing the changes in fluid saturation.

\subsection{Field development}

Facilities for managing fluid production and injection in block A were available by January 2010, when block A started production. When block B commenced production in January 2012, however, the facilities were upgraded to manage production and injection for the two blocks.

After blocks A and B had been in production for eight and a half and six and a half years, respectively, the assessment of the operator company was that although block A had been a success in terms of oil recovery and financial benefits, the opposite was true for block B, which had shown limited oil recovery, resulting in financial losses.

\section{Decision problem}

Next to block B, there is a third block (block C), with an area similar to that of blocks $\mathrm{A}$ and $\mathrm{B}$. Block $\mathrm{C}$ is separated from B by a north-south fault. The field operator needs to decide whether to continue with the development of the field towards block $\mathrm{C}$, or to restrict the reservoir development to the currently productive blocks, A and B.

\subsection{Problem alternatives}

The operator estimates that there are three options for continuing the development of the field and these are explored in the following sections.

\subsubsection{Without information}

This option entails the development of block $\mathrm{C}$, based on the current information. Facilities and flowlines will take 6 months to be ready and available, and the rig can be spud in 3 months, with another 3 months required to drill and complete the first well. Oil production with one well could start by January 2019, and another well will be added to the stream every 3 months. The full development will be complete with three producer wells in 6 months. Injector wells will be drilled when the producer wells are complete. The rig contract for the full six-well block will generate savings compared with drilling each well separately.

\subsubsection{With information}

The second option is to acquire additional information prior to deciding whether to develop block $\mathrm{C}$. The main uncertainty lies in the well's productivity, although well productivity has been shown to be related to the size of the OCT. It is believed that drilling an appraisal well in block $\mathrm{C}$ can unlock this project and generate the information necessary to decide whether the development of block $\mathrm{C}$ would be financially profitable.

It has been estimated that if the final decision is to develop block $\mathrm{C}$, the with information alternative will be delayed by 1 year compared to the without information alternative, due to the time needed to gather and analyse the data. In addition, the with information alternative will incur additional drilling costs due to the difference in the drilling contract strategies in both cases.

\subsubsection{Relinquish the development of block C}

The third option is to relinquish block $\mathrm{C}$ and only continue with the development of blocks A and B. This alternative will incur a loss of US\$34 million due to the fraction of the total cost of the reservoir development (seismic, offer, etc.) that has already been spent.

\section{Classical approach for VOI}

In this section of the paper, the classical approach for VOI is used to assess the best alternative to the decision problem described in Sect. 4.1.

As discussed in Sect. 3.2, the IOR in wells in block A ranges from 3200 to $5000 \mathrm{stb} / \mathrm{d}$, corresponding to wells of high productivity, while the IOR in wells in block B ranges from 20 to $250 \mathrm{stb} / \mathrm{d}$, corresponding to wells of low productivity. Based on geophysical and geological data and the IOR versus OCT correlation, it is estimated that the minimum IOR and maximum IOR for wells in block $\mathrm{C}$ are 20 and $5000 \mathrm{stb} / \mathrm{d}$, respectively, which will have consequences for the predicted $\mathrm{C}$ block profiles and the corresponding financial strength of this development.

For this analysis, there are three discrete production levels for block $\mathrm{C}$ : high-, medium- and low-production cases. The high case (state $s_{1}$ ) corresponds to the situation where the estimated IOR of block $\mathrm{C}$ is between 3200 and $5000 \mathrm{stb} / \mathrm{d}$, the low case (state $s_{3}$ ) corresponds to an IOR of between 20 and $250 \mathrm{stb} / \mathrm{d}$, and the medium case (state $s_{2}$ ) corresponds to an OCT of between 250 and $3200 \mathrm{stb} / \mathrm{d}$.

Due to the limited vertical area of each block and to avoid possible interference between the wells, blocks $\mathrm{A}$ and $\mathrm{B}$ include only three wells; this is also the number of wells planned for the development of block $\mathrm{C}$. 
The high-production case for block $\mathrm{C}$ is based on a threewell development, constructed using a well type which is an average of the well types in block A. The low-production case was built similarly, but using a well type which is an average of the well types in block B. The medium-production case is constructed as the arithmetic mean of the high and low cases; the use of this method is based on the linear correlation found between the OCT and the IOR.

\subsection{Without information}

In the option where the project moves to the development phase without data acquisition, there are three potential production profiles for block $\mathrm{C}$, corresponding to the high, low and medium cases, as shown in Fig. 4. These cases correspond to the chances of finding: (1) "high-IOR" wells or similar to what was found in block A, (2) "low-IOR" wells or similar to what was found in block B or, (3) "medium IOR" wells or at an average value between what was found in blocks A and B. The prior probability assigned to each state of nature (for each case) is based on the experts' judgment of the likelihood of realising that state. The value of each state is equal to the net present value of the project associated with the corresponding profile (high, medium and low), each with a 20 -year horizon. Table 1 shows the prior probabilities that were assigned, and the net present values calculated for each state of nature.

Based on the prior probabilities and values shown in Table 2 and using Eqs. (3) and (4) in Sect. 2, the EV of this project is estimated to be US\$261 million for the without information option.

\subsection{With information}

When assessing the with information option, the objective is to estimate the value added to the project from the

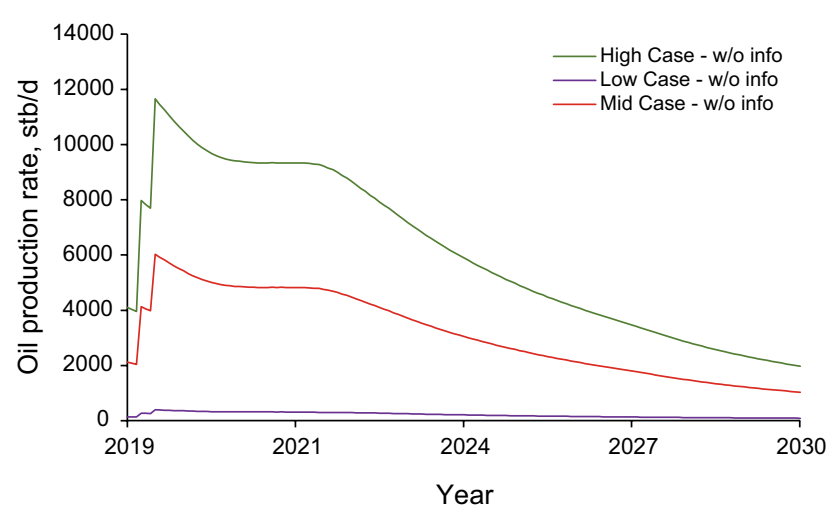

Fig. 4 Predicted profiles for the three states of nature in the without information option. "w/o info" means the case without new information
Table 2 Prior probabilities and values for the three states of nature

\begin{tabular}{llc}
\hline State of nature & Prior probability (fraction) & $\begin{array}{l}\text { Value, } \\
\text { US\$ mil- } \\
\text { lion }\end{array}$ \\
\hline$s_{1}=$ high & 0.25 & 636 \\
$s_{2}=$ medium & 0.50 & 263 \\
$s_{3}=$ low & 0.25 & -119 \\
\hline
\end{tabular}

additional information gathered. To assess the value of acquiring new data, the range of possible outcomes for OCT $(11-47 \mathrm{ft})$ is discretised into 12 intervals, each being $3 \mathrm{ft}$ in length.

The reliability probability (the conditioned probability that the data outcome accurately predicts the state of nature) is assigned by the expert members of the technical team, based on their knowledge of the geological setting and previous exploration activities in the area or in similar settings. The reliability probabilities assigned by the expert members of the technical team for this case study are shown in Table 3. Figure 5 displays the reliability probability used in this assessment, where the overlapping curve is characteristic of the imperfect nature of the data.

These reliability probabilities cannot be used directly in the VOI assessment and Bayes' theorem is thus used to flip the reliability probabilities to obtain the posterior probabilities (the probabilities of each state of nature conditioned to each possible outcome of the data acquisition), which are then used in the VOI assessment.

One additional probability is computed during the application of Bayes' theorem, and this is associated with realising each of the outcomes from the data acquisition and is known as the marginal probability. Table 4 shows the posterior probabilities, marginal probabilities and EV.

Using the data in Table 4 and Eqs. (9)-(11), the EV of this project (with information) is estimated to be US\$267 million.

The project's value for the relinquish alternative (A2) is - US\$34 million. This means that $\operatorname{EV}\left(A_{2} \mid x_{k}\right)$ is - US\$34 million for all the values of $x_{k}$.

A comparison of the values of the project with and without information shows that there is a total positive gain in acquiring information. Using Eq. (12), the VOI is estimated to be US\$6 million. Based on this assessment, it is recommended that new data should be acquired before developing block $\mathrm{C}$.

This means that, of the three alternatives evaluated, the optimal choice is for data acquisition, although this is only minimally better than the alternative of continuing the project without acquiring new data. 
Table 3 Reliability probabilities showing the mid-value of each interval

\begin{tabular}{lllllll}
\hline & $x_{1}=45$ & $x_{2}=42$ & $x_{3}=39$ & $x_{4}=36$ & $x_{5}=33$ & 0.100 \\
\hline$p\left(x_{k} \mid s_{1}\right)$ & 0.250 & 0.250 & 0.230 & 0.180 & 0.200 & 0.000 \\
$p\left(x_{k} \mid s_{2}\right)$ & 0.000 & 0.000 & 0.000 & 0.050 & 0.250 \\
$p\left(x_{k} \mid s_{3}\right)$ & 0.000 & 0.000 & 0.000 & $x_{9}=21$ & $x_{10}=18$ & $x_{11}=15$ \\
\hline & $x_{7}=27$ & $x_{8}=24$ & 0.000 & 0.000 & 0.000 \\
\hline$p\left(x_{k} \mid s_{1}\right)$ & 0.000 & 0.000 & 0.000 & 0.000 & 0.000 \\
$p\left(x_{k} \mid s_{2}\right)$ & 0.250 & 0.200 & 0.180 & 0.230 & 0.250 \\
$p\left(x_{k} \mid s_{3}\right)$ & 0.000 & 0.100 & & 0.000 & 0.250 \\
\hline
\end{tabular}

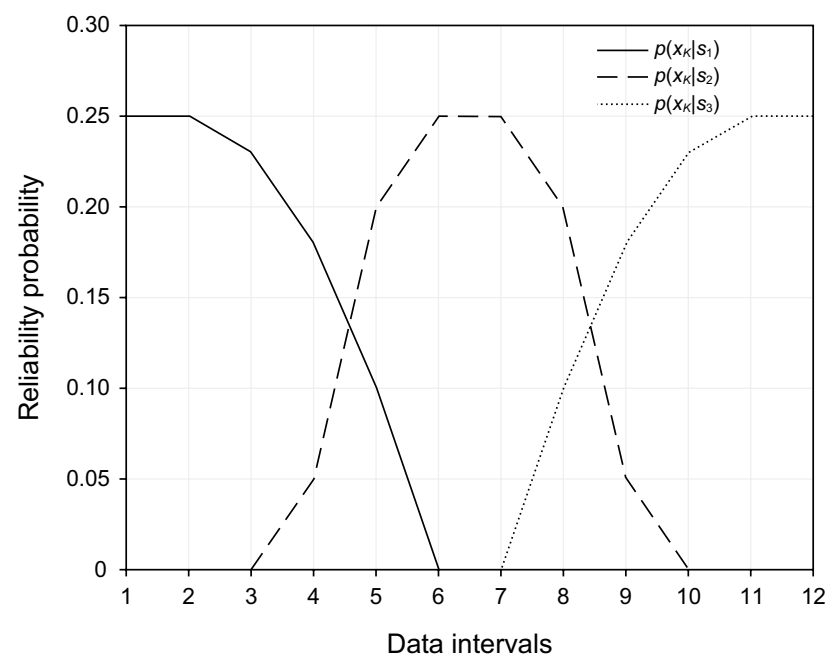

Fig. 5 Reliability probability assigned by technical experts

\section{Application of fuzzy logic approach for VOI}

The VOI discussed in Sect. 3.2 assumes that the outcomes of OCT measurements are crisp values. For the decision problem under discussion, crisp measurements assume that:

(1) Saturation $\log s$ developed from the electrical response and fluid density data from RFT pressure tests carried out on the appraisal well in block $\mathrm{C}$ will clearly define the location of the oil-water contact and the transition zone.

(2) The top of the structure in block C is well defined and, with the proposed appraisal well tied to the structure, it will be possible to accurately describe the structure of the rest of block C. In these blocks, oil is undersaturated and no gas cap is expected, meaning that the

Table 4 Posterior probabilities, marginal probabilities and EVs

\begin{tabular}{|c|c|c|c|c|c|c|}
\hline & $x_{1}=45$ & $x_{2}=42$ & $x_{3}=39$ & $x_{4}=36$ & $x_{5}=33$ & $x_{6}=30$ \\
\hline$p\left(s_{1} \mid x_{k}\right)$ & 1.000 & 1.000 & 1.000 & 0.643 & 0.2000 & 0.000 \\
\hline$p\left(s_{2} \mid x_{k}\right)$ & 0.000 & 0.000 & 0.000 & 0.357 & 0.8000 & 1.000 \\
\hline$p\left(s_{3} \mid x_{k}\right)$ & 0.000 & 0.000 & 0.00 & 0.000 & 0.0000 & 0.000 \\
\hline$p\left(x_{k}\right)$ & 0.063 & 0.063 & 0.058 & 0.070 & 0.125 & 0.125 \\
\hline$E V\left(A_{1} \mid x_{k}\right)$ & 621 & 621 & 621 & 488 & 324 & 250 \\
\hline$E V\left(A_{2} \mid x_{k}\right)$ & -34 & -34 & -34 & -34 & -34 & -34 \\
\hline \multirow[t]{2}{*}{$E V\left(* \mid x_{k}\right)$} & 621 & 621 & 621 & 488 & 324 & 250 \\
\hline & $x_{7}=27$ & $x_{8}=24$ & $x_{9}=21$ & $x_{10}=18$ & $x_{11}=15$ & $x_{12}=12$ \\
\hline$p\left(s_{1} \mid x_{k}\right)$ & 0.000 & 0.000 & 0.000 & 0.000 & 0.000 & 0.000 \\
\hline$p\left(s_{2} \mid x_{k}\right)$ & 1.000 & 0.800 & 0.357 & 0.000 & 0.000 & 0.000 \\
\hline$p\left(s_{3} \mid x_{k}\right)$ & 0.000 & 0.200 & 0.643 & 1.000 & 1.000 & 1.000 \\
\hline$p\left(x_{k}\right)$ & 0.125 & 0.125 & 0.070 & 0.058 & 0.063 & 0.063 \\
\hline$E V\left(A_{1} \mid x_{k}\right)$ & 250 & 175 & 11 & -122 & -122 & -122 \\
\hline$E V\left(A_{2} \mid x_{k}\right)$ & -34 & -34 & -34 & -34 & -34 & -34 \\
\hline$E V\left(* \mid x_{k}\right)$ & 250 & 175 & 11 & -34 & -34 & -34 \\
\hline
\end{tabular}


Table 5 Membership function values for 12 fuzzy intervals

\begin{tabular}{lllllll}
\hline & $x_{1}=45$ & $x_{2}=42$ & $x_{3}=39$ & $x_{4}=36$ & $x_{5}=33$ & $x_{6}=30$ \\
\hline$\mu\left(\tilde{M}_{1} \mid x_{k}\right)$ & 0.75 & 0.73 & 0.67 & 0.55 & 0.28 & 0.10 \\
$\mu\left(\tilde{M}_{2} \mid x_{k}\right)$ & 0.15 & 0.15 & 0.18 & 0.25 & 0.44 & 0.28 \\
$\mu\left(\tilde{M}_{3} \mid x_{k}\right)$ & 0.10 & 0.12 & 0.15 & 0.20 & $x_{11}=15$ & 0.33 \\
\hline & $x_{7}=27$ & $x_{8}=24$ & $x_{9}=21$ & 0.00 & 0.00 & 0.12 \\
$\mu\left(\tilde{M}_{1} \mid x_{k}\right)$ & 0.02 & 0.00 & 0.00 & 0.30 & 0.80 \\
$\mu\left(\tilde{M}_{2} \mid x_{k}\right)$ & 0.61 & 0.59 & 0.44 & 0.70 & 0.10 \\
$\mu\left(\tilde{M}_{3} \mid x_{k}\right)$ & 0.37 & 0.41 & 0.56 & & 0.90 \\
\hline
\end{tabular}

upper limit of the oil column is at the top of the structure.

However, OCT data based on saturation logs and RFTs carry a great deal of ambiguity and no clear value was measured for any of the wells in blocks A and B. The same situation is expected in block $\mathrm{C}$.

In addition, there is uncertainty in the definition of the top of the structure. The seismic data are of medium quality, with an estimated vertical resolution of between 10 and $15 \mathrm{ft}$. Even after the appraisal well in block $\mathrm{C}$ is tied to the structure, the remaining wells to be drilled in block $\mathrm{C}$ are expected to show deviation with respect to the structure (the same situation holds for blocks A and B).

The factors discussed above mean that the data to be acquired are inherently vague or diffuse, and this justifies the use of fuzzy concepts in the VOI assessment. Following this reasoning, fuzzy logic is applied to the assessment of the reliability and posterior probabilities, in order to handle the fuzzy nature of the data assessed in the VOI.

\subsection{Fuzzy data acquisition}

The case study described in Sect. 2 is used for the analysis in this section. Three fuzzy events for the OCT are considered: large OCT $\left(\tilde{M}_{1}\right)$, medium OCT $\left(\tilde{M}_{2}\right)$ and low $\operatorname{OCT}\left(\tilde{M}_{3}\right)$.

The membership functions describe the "degree of belonging" of each interval to each of the fuzzy events, as shown in Table 5. Figure 6 presents the shape of the curves that describe the membership functions used in this assessment.

The shapes of the membership functions in Fig. 6 show the ambiguity of the data to be acquired, according to expert opinion. In a fuzzy description of the data to be gathered, each of the 12 intervals within the range of possible outcomes belongs to one or more of the membership functions. Due to the fuzziness in the data, a given outcome not only belongs to the membership function $\mu\left(\tilde{M}_{1} \mid x_{k}\right)$ but also belongs to the membership function $\mu\left(\tilde{M}_{2} \mid x_{k}\right)$. The degree of belonging is different for each

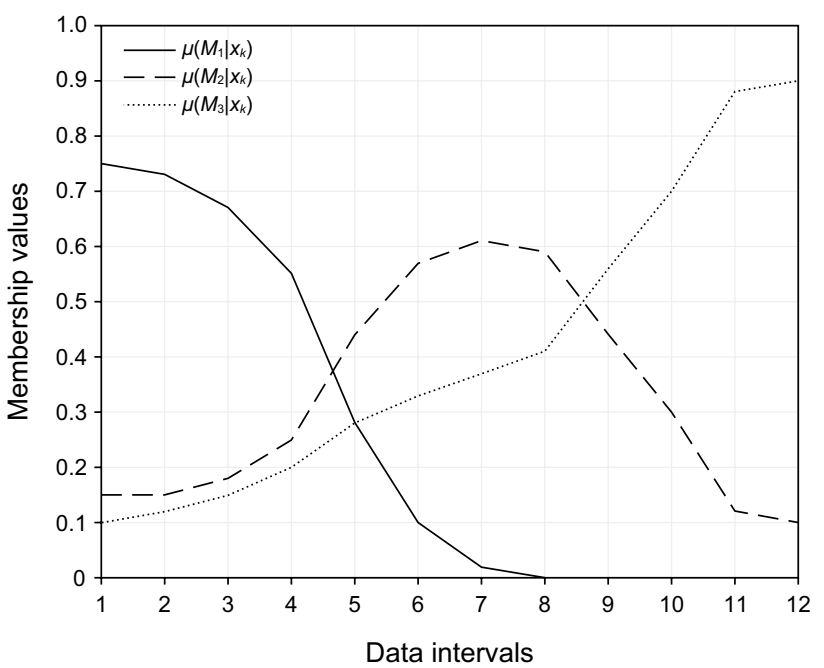

Fig. 6 Membership functions used in the assessment

Table 6 Fuzzy reliability probabilities

\begin{tabular}{llll}
\hline & $s_{1}$ & $s_{2}$ & $s_{3}$ \\
\hline$p\left(\tilde{M}_{1} \mid s_{k}\right)$ & 0.651 & 0.114 & 0.000 \\
$p\left(\tilde{M}_{2} \mid s_{k}\right)$ & 0.205 & 0.536 & 0.262 \\
$p\left(\tilde{M}_{3} \mid s_{k}\right)$ & 0.154 & 0.351 & 0.748 \\
\hline
\end{tabular}

membership function, and a similar situation holds for the other outcomes and membership functions. In general, the membership functions were constructed on the assumption that when high values of OCT are reported, these values have a degree of belonging not only to the "large" OCT membership function but also to the "medium" function. However, when low values of OCT are reported, the data primarily belong to the "low" membership function.

Using Eqs. (17) and (18) and the values in Tables 3 and 5 , the fuzzy reliability probabilities and the fuzzy posterior probabilities are computed, and the results are presented in Tables 6 and 7. 
Table 7 Fuzzy posterior probabilities

\begin{tabular}{llll}
\hline & $\tilde{M}_{1}$ & $\tilde{M}_{2}$ & $\tilde{M}_{3}$ \\
\hline$p\left(s_{1} \mid \tilde{M}_{k}\right)$ & 0.741 & 0.133 & 0.096 \\
$p\left(s_{2} \mid \tilde{M}_{k}\right)$ & 0.259 & 0.696 & 0.438 \\
$p\left(s_{3} \mid \tilde{M}_{k}\right)$ & 0.000 & 0.170 & 0.466 \\
\hline
\end{tabular}

Table 8 Expected fuzzy values

\begin{tabular}{lccc}
\hline & $\tilde{M}_{1}$ & $\tilde{M}_{2}$ & $\tilde{M}_{3}$ \\
\hline $\operatorname{EV}\left(A_{1} \mid \tilde{M}_{k}\right)$ & 525 & 236 & 112 \\
$\operatorname{EV}\left(A_{2} \mid \tilde{M}_{k}\right)$ & -34 & -34 & -34 \\
\hline
\end{tabular}

The EVs of both alternatives are calculated for each fuzzy interval using Eq. (20), and the results are summarised in Table 8.

Finally, using Eqs. (21) and (22) in Sect. 2 and the values given in Tables 7 and 8, the expected fuzzy value for the with information alternative is estimated to be US\$251 million.

The VOI for the project with fuzzy data acquisition is estimated to be - US\$10 million, using Eq. (23) in Sect. 2. Based on this assessment, it is not recommended that new data be acquired before developing block $\mathrm{C}$ since the VOI for fuzzy data is lower than the corresponding value for the case where crisp data were assumed.

\section{Results}

When using the classical approach for VOI, the EV for the projects is US\$261 million for the no data acquisition, - US\$34 million for the relinquish case and US\$267 million for the data acquisition alternative. Based on this assessment, the recommended decision is to acquire the data and use the outcome of the data acquisition to decide whether or not to sanction the project. When using the fuzzy data approach for VOI, the value of the projects is still US $\$ 261$ million for the no data acquisition, - US\$34 million for the relinquish alternative and US\$251 million for the data acquisition alternative. Based on this approach, the recommended decision is to not acquire the data and to sanction the project with the existing information.

The results of fuzzy VOI assessments show that the value of the data decreases when it is integrated into the assessment compared with the standard VOI. In this case study, the value of the project with information decreases from US\$267 million to US\$251 million; the decrease in value is enough to change the decision from with information to without information, which has a value of US\$261 million.
The impact (decision change) of including the imprecision of the data in the assessment depends on the membership functions used and the difference between the with and without information alternatives. As a rule, when the data to be acquired are imprecise, it is recommended to use fuzzy VOI methodology.

The fuzziness of the data reduces the impact that it has on the project's value; if the fuzzy nature of the data is not included in the assessment, VOI can result in an optimistic assessment of the value of the data, which can lead to bad decisions. Including the fuzzy nature of the data in the VOI methodology secures a more accurate assessment of the data and consequently a better decision process.

\section{Conclusions}

In this paper, the crisp and fuzzy theoretical formulations of VOI are described. The main difference between them is that the fuzzy formulation uses membership functions to represent the imprecision associated with the data that will be gathered, while the classical formulation assumes that the data are crisp. In the fuzzy approach, membership functions are used in conjunction with Bayes' theorem in order to convert reliability probabilities into the posterior probabilities.

A case study of an oil and gas development project is discussed, in which both crisp and fuzzy VOI methodologies are applied to a subsurface data acquisition problem for which the data are inherently vague. The contrasting results of these two assessments suggest that when the data are fuzzy, a fuzzy approach to evaluating VOI should be used in order to produce a correct assessment of the suitability of acquiring such data.

In this case study, the fuzzy assessment assigns a smaller value to the with information alternative compared to that of the crisp assessment. The consideration of the fuzzy nature of the new data to be acquired therefore has an impact on the VOI assessment.

The analysis carried out in this paper shows that the outcomes of the VOI assessment depend on the formulation used, i.e. crisp or fuzzy. The selection of the formulation should therefore take into consideration the nature of the data to be acquired.

Thus, the main contributions of this work can be summarised as follows:

- It is shown that there are situations in the oil and gas industry in which the VOI is affected not only by the uncertainty associated with a lack of knowledge of the project input variables but also by the imprecision associated with the outcomes of the data to be acquired;

- The use of fuzzy data modelling is proposed to handle the data imprecision in the VOI assessment; 
- A practical application of this methodology is demonstrated using a case study of an oil and gas project.

Future work will address the problem of applying fuzzy inference systems in the assessment of VOI.

Open Access This article is distributed under the terms of the Creative Commons Attribution 4.0 International License (http://creativeco mmons.org/licenses/by/4.0/), which permits unrestricted use, distribution, and reproduction in any medium, provided you give appropriate credit to the original author(s) and the source, provide a link to the Creative Commons license, and indicate if changes were made.

\section{References}

Ahmed A, Elkatatny S, Ali A, et al. Rate of penetration prediction in shale formation using fuzzy logic. In: International technology conference. Beijing, China, 26-28 March 2019. IPTC-19548-MS. https://doi.org/10.2523/iptc-19548-ms.

Begg S, Bratvold R, Campbell J. The value of flexibility in managing uncertainty in oil and gas investments. In: Society of petroleum engineers annual technical conference and exhibition. San Antonio, Texas, USA, 29 Sept-2 Oct 2002. SPE 77586. https://doi. org/10.2118/77586-ms.

Bermudez F, Carvajal G, Moricca G, et al. Fuzzy logic application to monitor and predict unexpected behavior in electric submersible pumps. In: Society of petroleum engineers. Intelligent energy conference and exhibition. Utrecht, the Netherlands, 1-3 April 2014. SPE 167820-MS. https://doi.org/10.2118/167820-ms.

Bickel J. Decision analytics, value of information and piloting in unconventional reservoirs. In: Society of petroleum engineers. Hydrocarbon Economics and Evaluation Symposium. Houston, Texas, USA. 19-20 May 2014. SPE 169841-MS. https://doi. org/10.2118/169841-ms.

Bratvold R, Bickel J, Lohne H. Value of Information in the oil and gas industry: past, present, and future. In: Society of petroleum engineers annual technical conference and exhibition. Anaheim, California, USA, 11-14 Nov 2007. SPE 110378. https://doi. org/10.2118/110378-ms.

Bukhamseen N, Al-Najem A, Saffar A, et al. Streamline information guided by fuzzy logic to optimize field injection/production strategies. In: Society of petroleum engineers. Kingdom of Saudi Arabia technical symposium and exhibition. Dammam, Saudi Arabia, 25-28 April 2016. SPE 182742-MS. https://doi. org/10.2118/182742-ms.

Clemen R. Making hard decisions. 2nd ed. California: Duxbury Press; 1996.

Coopersmith E, Cunningham P. A practical approach to evaluating the value of information and real option decisions in the upstream petroleum industry. In: Society of petroleum engineers, annual technical conference and exhibition, San Antonio, Texas, USA, 29 Sept-2 Oct 2002. SPE 159587. https://doi.org/10.2118/77582-ms.

Demirmen F. Use of value of information concept in justification and ranking of subsurface appraisal. In: Society of petroleum engineers, annual technical conference and exhibition. Denver, Colorado, USA, 6-9 Oct 1996. SPE 36631. https://doi. org/10.2118/36631-ms.

Ebrahimi E, Sajedian A. Use of fuzzy logic for predicting two-phase inflow performance relationship of horizontal oil wells. In: Society of petroleum engineers. Trinidad and Tobago energy resources conference. Port Spain, Trinidad, 27-30 June 2010. SPE 133436. https://doi.org/10.2118/133436-ms.
Ferreira C. A probabilistic approach to quantify the value of information of 4D seismic projects. In: Society of petroleum engineers. Annual technical conference and exhibition. Houston, Texas, USA, 28-30 Sept 2015. SPE 178731-STU. https://doi. org/10.2118/178731-stu.

Grassian D, Bahatem M, Scott T, et al. Application of a fuzzy expert system to analyse and anticipate ESP failure modes. In: Society of petroleum engineers. Abu Dhabi international petroleum exhibition and conference. Abu Dhabi, UAE, 13-16 Nov 2017. SPE 188305-MS. https://doi.org/10.2118/188305-ms.

Grayson C. Decision under uncertainty: drilling decisions by oil and gas operators. Boston: Division of Research, Graduate School of Business, Harvard University; 1960. ISBN 0875840159

Grose T, Smalley P. Risk based surveillance planning: a practical value of information approach for data acquisition in producing fields. In: Society of petroleum engineers. January 2017 SPE economics and management. SPE 184409. p. 1-11. https://doi. org/10.2118/184409-pa.

Hayward G, Davidson V. Fuzzy logic applications. Analyst. 2003;128:1304-6. https://doi.org/10.1039/b312701j.

Howard R. Decision analysis: applied decision theory. In: Hertz DB, Melese J, editors. Proceedings of the fourth international conference on operational research. New York: Wiley; 1966. https ://doi.org/10.2307/3008168.

Ivanovich K, Evgenevich K, Sergeevich M. Increase of certainty of calculation of hydrocarbon reserves on the basis of fuzzy petrophysical compositions method. In: Society of petroleum engineers. Russian oil and gas exploration and production technical conference and exhibition. Moscow, Russia, 16-18 Oct 2012. SPE 162038. https://doi.org/10.2118/162038-ms.

Koninx J. Value-of-information - from cost cutting to value-creation. In: Society of petroleum engineers, Asia Pacific oil and gas conference and exhibition. Brisbane, Australia, 16-18 Oct 2000. SPE 64390. https://doi.org/10.2118/64390-ms.

Kullawan K, Bratvold R, Bickel J. Value creation with multi-criteria decision making in geosteering operations. In: Society of petroleum engineers hydrocarbon economics and evaluation symposium. Houston, Texas, USA, 19-20 May 2014. SPE 169849-MS. https://doi.org/10.2118/169849-ms.

Lohrenz J. Net values of our information. In: Journal of petroleum technology, society of petroleum engineers; 1988. p. 499-503. SPE 16842. https://doi.org/10.2118/16842-pa.

Mirzabozorg A, Nghiem L, Chen Z, et al. How does the incorporation of engineering knowledge using fuzzy logic during history matching impact reservoir performance prediction? In: Society of petroleum engineers. Heavy oil conference. Alberta, Canada, 10-12 June 2014. SPE 170011-MS. https://doi. org/10.2118/170011-ms.

Nageh M, Abu M, El Tayeb E, et al. Application of using fuzzy logic as an artificial intelligence technique in screening criteria of the EOR technologies. In: Society of petroleum engineers. North Africa technical conference and exhibition. Cairo, Egypt, 14-16 Sept 2015. SPE 175883-MS. https://doi.org/10.2118/175883-ms.

Nashawi I, Malallah A. Permeability prediction from wireline well $\operatorname{logs}$ using fuzzy logic and discriminant analysis. In: Society of petroleum engineers. Asia pacific oil and gas conference and exhibition. Brisbane, Queensland, Australia, 18-20 Oct 2010. SPE 133209. https://doi.org/10.2118/133209-ms.

Newendorp P. Application of utility theory to drilling investment decisions. Ph.D. thesis, 1967, ed. Department of Engineering, University of Oklahoma, USA. https://shareok.org/bitstream/handl e/11244/2247/6715898.PDF?sequence=1\&isAllowed=y.

Newendorp P, Schuyler J. Decision analysis for petroleum exploration. 2nd ed. Aurora: Planning Press; 2002. ISBN 978-0966440119. 
Okuda T. A formulation of fuzzy decision problems with fuzzy information using probability measures of fuzzy events. Inf Control. 1978;38:135-47. https://doi.org/10.1016/s0019-9958(78)90151-1.

Passalacqua H, Qubian A. Improving the decision making for heavy oil field development projects using the fuzzy analytical hierarchy process FAHP. In: Society of petroleum engineers. International heavy oil conference and exhibition. Kuwait City, Kuwait, 10-12 Dec 2018. SPE 193719-MS. https://doi.org/10.2118/193719-ms.

Popa A. Identification of horizontal well placement using fuzzy logic. In: Society of petroleum engineers. Annual technical conference and exhibition. New Orleans, Louisiana, USA, 30 Sept-2 Oct 2013. SPE 166313. https://doi.org/10.2118/166313-ms.

Raiffa H. A personal account of how it got started and evolved. In: Advances in decision analysis. Cambridge University Press; 2007. p. 1-17. https://doi.org/10.1287/opre.50.1.179.17797.

Raiffa H, Schlaifer R. Applied statistical decision theory. 3rd ed. Boston: Division of Research, Graduate School of Business Administration, Harvard University; 1961. https://www.gwern .net/docs/statistics/decision/1961-raiffa-appliedstatisticaldecisio ntheory.pdf.

Ross T. Fuzzy logic with engineering applications. 3rd ed. West Sussex: Wiley; 2010. https://doi.org/10.1002/9781119994374.

Sari M. Estimating strength of rock masses using fuzzy inference system. In: Ulusay R et al, editors. Rock mechanics and rock engineering: from the past to the future. London: Taylor \& Francis Group; 2016. ISBN 978-1-138-03265-1. https://doi. org/10.1201/9781315388502-20.

Shabair A, Hammadi A, Adeoye O, et al. The value of reservoir surveillance applications to fracture carbonates under waterflooding. In:
Society of petroleum engineers. Abu Dhabi international petroleum exhibition and conference. Abu Dhabi, UAE, 13-16 Nov 2017. SPE 188192-MS. https://doi.org/10.2118/188192-ms.

Sivanandam S, Sumathi S, Deepa S. Introduction to fuzzy logic using MATLAB. Heidelberg: Springer; 2010. https://doi. org/10.1007/978-3-540-35781-0.

Steineder D, Clemens T, Osivandi K, et al. Maximizing value of information of a horizontal polymer pilot under uncertainty. In: Society of petroleum engineers. 80th EAGE conference and exhibition. Copenhagen, Denmark, 11-14 June 2018. SPE 190871-MS. https ://doi.org/10.2118/190871-ms.

Suslick S, Schiozer D. Risk analysis applied to petroleum exploration and production: an overview. J Pet Sci Eng. 2004;44(2):1-9. https ://doi.org/10.1016/j.petrol.2004.02.001.

Thong D, Kepic A. Incorporating information into seismic impedance inversion using fuzzy clustering technique. In: SEG New Orleans annual meeting; 2015. p. 3451-5. https://doi.org/10.1190/segam 2015-5922589.1.

Warren J. The development decision: value of information. In: American Institute of Mining, Metallurgical and Petroleum Engineers. Society of petroleum engineers hydrocarbon economics and evaluation symposium of the society of petroleum engineers. Dallas, Texas, USA, March 3-4 1983. SPE 11312. https://doi. org/10.2118/11312-ms.

Zadeh L. Fuzzy sets. Inf Control. 1965;8:338-52. https://doi. org/10.1016/s0019-9958(65)90241-x.

Zadeh L. Probability measure of fuzzy events. J Math Anal Appl. 1968;23:421-7. https://doi.org/10.1016/0022-247x(68)90078-4. 